\title{
TELECONSUlTATION WiTH A DEVELOPING COUNTRY: STUDENT REPORTED OUTCOMES OF LEARNING
}

\author{
MEGAN K. FOTI, DOT, MS, OTR, CRYSTAl ElEAZAR, BS, OTS, \\ KIMBERLY A. FURPHY, DHSC, OT, ATP \\ The Richard Stockton COllege Of NeW Jersey, Galloway, NJ, USA
}

ABSTRACT

This qualitative study explored the benefits of implementing (international) teleconsultation in a Master of Science in Occupational Therapy (MSOT) curriculum. Twenty-one students provided supervised teleconsultative services to individuals with disabilities in Guatemala and were responsible for completing assessments, setting goals, and providing resources to address goals and improve quality of life. Data were collected through student presentations and coded for relevant themes. Analysis revealed new learning in the areas of the occupational therapy process, cultural awareness, and technology. Three themes emerged: Increased Understanding of Awareness of and Challenges to Working with People of a Different Culture; Need for Adaptability and Flexibility as Practicing Clinicians; Emerging Role of Technology in Occupational Therapy. Based on results from this study, occupational therapy academicians should consider implementing similar programs into curricula and conduct related research in order to promote not only student learning, but also to advance the use of telehealth technology in occupational therapy practice.

Key words: Guatemala, occupational therapy, teleconsultation, telehealth

Occupational therapy education poses many challenges for educators, one of which is structuring a learning environment that promotes collaborative learning, critical thinking, interactive learning experiences and increased student engagement. Additionally, the American Occupational Therapy Association's Accreditation Council of Occupational Therapy Education (ACOTE®) and Centennial Vision place certain expectations on

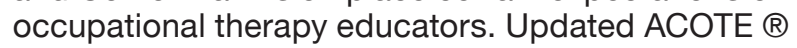
standards (2011) require the integration of technology into curricula (B.1.8), which may include telehealth, the provision of remote services via technology. The Centennial Vision of the American Occupational Therapy Association (AOTA, 2013) tasks occupational therapy practitioners and educators to address global needs. In order to enrich student learning and meet the aforementioned expectations, a Master of Science in Occupational Therapy (MSOT) program integrated telehealth into the MSOT curriculum with positive outcomes. Faculty mentored students in the use of telehealth technologies to provide remote services (i.e., teleconsultation) to clients in Guatemala. This article will describe the pedagogy and outcomes associated with integrating telehealth into the MSOT curriculum.

Telehealth is a service delivery model whereby health related services are provided at a distance via telecommunications technologies. AOTA (2013) defines telehealth as "the application of evaluative, consultative, preventative, and therapeutic services delivered through telecommunication and information technologies" (p. S69). A primary benefit of providing services via telehealth technology is improved access to health related services and specialists for individuals living in rural or underserved areas (AOTA, 2013). There is a growing body of evidence supporting the use of telehealth as an effective service delivery model in occupational therapy (AOTA, 2013; Barlow, Liu, \& Sekulic, 2009; Cason, 2009, 2011; Hoffmann \& Russell, 2008; Hoffmann, Russell, Thompson, Vincent, \& Nelson, 2008; Jacobs, Blanchard, \& Baker, 2012; Schein et al., 2011; Sanford et al., 2007). In addition to being an effective service delivery model, the integration of telehealth in academia enhances the educational experience and facilitates confidence and competence in the use of telehealth technologies among future healthcare practitioners (Miller et al., 2003).

Telehealth in the classroom presents educators with an opportunity to utilize technology as a way of promoting an advanced learning environment and as a means of expanding the provision of "high-quality, efficient health care education" (Lee et al., 2012, p. 90). Bulik and Shokar (2010) reported on seven fourth-year medical students who participated in a month-long elective telemedicine 
course to learn about telehealth technologies and to explore providers' and patients' perspectives of the service delivery model. Students were paired with physicians providing telemedicine and assisted with remote interviews and physical examinations with patients. During the latter part of the elective course, students interviewed patients to obtain their perspective of the patient-physician relationship that developed via electronic means. Students provided course feedback (i.e., reflective essays) and articulated the importance of learning about and incorporating telehealth into their future practice (Bulik \& Shokar, 2010).

Similarly, undergraduate, graduate, and clinical educators and providers who participated in a telehealth program at the University of Alberta from 1996-1999 provided instrumental perspectives on the benefits of utilizing telehealth. The authors indicated that the program facilitated experiences with a variety of interdisciplinary placements and specialized rehabilitation services. Additionally, students had easier access to education and participation in special interest groups (Liu \& Miyazaki, 2000).

While evidence supporting the use of telehealth in occupational therapy continues to expand, current evidence supporting the pedagogical outcomes from allied health students' participation in a teleconsultation program is limited. Two faculty members at the institution aligned a teleconsultation project with an adult interventions course in order to address occupational therapy education accreditation standards and enrich student learning. Students were required to provide a brief presentation on their experience and outcomes of the teleconsultation project. The purpose of the study was to evaluate the students' learning and the pedagogical benefits of implementing (international) teleconsultation in an MSOT interventions course. It was hypothesized that as a result of international service learning and teleconsultation, students would demonstrate increased role competence (Fries et al., 2013; Lee, 2009).

\section{METHODOLOGY}

\section{PRELIMINARY STUDY}

In a related preliminary study conducted by graduate students in the highlighted MSOT program titled, Facilitators and Barriers to Living with a Disability in Guatemala, investigators used a phenomenological study design to understand the lived experience of someone with a disability in Guatemala (Eleazar, Campisi, \& Foti, 2013). This single case study was conducted utilizing a semi-structured, open-ended interview with a 32 year old male living in Guatemala who sustained a C5 incomplete spinal cord injury approximately four years prior to the study. The interview was video and audio recorded, transcribed, and coded for emerging themes.

Results from this study yielded information on facilitators and barriers to living with a disability in a developing country and included cultural, social, and political information about Guatemala, especially as it relates to healthcare and receiving rehabilitation services such as occupational and physical therapy. Healthcare services in Guatemala including rehabilitation are expensive, which is a significant barrier to accessing and receiving care. Cost of therapy can range anywhere from $\$ 250-\$ 300$ (USD) per 20 minute session as access to managed healthcare is limited and difficult to acquire. Oftentimes, when healthcare services are available, the quality of care is limited. As noted by our participant, it can take up to one to two months to receive results for a standard x-ray.

Environmental factors such as uneven sidewalks and lack of public transportation can be problematic to individuals living with a disability in developing countries. In Guatemala specifically, the homeowners are responsible for designing and creating a sidewalk in front of their house, if they choose to do so. There are no requirements for sidewalks to be even, therefore the height and design of the sidewalks varies from house to house, thus further limiting independence with community mobility. Public transportation is not available in many areas, and if it is, most vehicles are not equipped to accommodate individuals who have disabilities.

Social barriers create a more challenging environment to accessing care. The study participant indicated there is often a stigma associated with individuals living with a disability.

"Here, they see a person in a wheelchair like something is different....maybe they think we're not the same, normal person, like a regular person...so it's very hard to get a job. People with disabilities, they always stay in the house." (Eleazar, Campisi, \& Foti, 2013).

This stigma contributes to ongoing levels of poverty, which in turn limit an individual's access to benefits, and ultimately care.

Results and insight from this study were used by student investigators to create an informational presentation for peers who would be participating in the teleconsultation experience. Researchers shared results in order to provide relevant background information and increase cultural awareness. After this information was disseminated to student groups, they were paired with a person with a disability in Guatemala in order to participate in an experiential learning assignment.

\section{Curricular Design}

Twenty-one MSOT students were assigned to groups of three and collaborated with community occupational therapists in order to provide teleconsultation services to individuals with disabilities in Guatemala. Students 
were charged with the tasks of completing assessments, identifying areas of impairment, setting goals, and providing their clients with ideas and resources to address goals and improve quality of life. At the Guatemala clinic associated with the study, a physical therapist that was providing services to the clients was asked to facilitate implementation of the recommendations, as appropriate. Utilizing telehealth in combination with international learning experiences, this MSOT program yielded many opportunities for advancing student learning and competency. However, in order to investigate how this experience influenced learning, it was essential to collect and review student feedback.

Students were provided with an assignment outline and grading rubric in order to highlight expectations (K. Furphy, personal communication, December 6, 2013) (See Appendices $\mathrm{A}$ and $\mathrm{B}$ for assignment outline and grading rubric).

\section{PARTICIPANTS}

Participants in this study were 21 graduate students enrolled in their third semester of a two and a half year occupational therapy program. The participants were (a) ages 22-37 years; (b) female $(n=19)$ and male $(n=2)$; and (c) enrolled in "Adult Interventions," a graduate level occupational therapy course which included a pilot program that provided teleconsultation services to individuals in Guatemala. Participation was voluntary and all participants signed an institutionally reviewed and approved consent form.

\section{DATA COLlECTION}

Data were collected during student group presentations lasting between ten and twenty minutes. The assigned presentations required students to share their experiences as per the assignment guideline (Appendix A).

Presentations were videotaped and all audio was transcribed verbatim by a graduate research assistant who did not participate in the teleconsultation assignment.

\section{DATA ANALYSIS}

Data analysis, which was done manually, began after all presentations were transcribed. The primary investigator and two research assistants individually reviewed a qualitative rubric and coded data into relevant themes.

First, using the qualitative rubric, levels of new learning were investigated in the outcome areas of: the occupational therapy process, cultural awareness, and understanding of the role of technology in the occupational therapy profession. Using only explicit statements made by participants, feedback was ranked 0-3 based on how many areas of learning (i.e., occupational therapy process, culture, and technology) were reported. A score of " 0 " indicated no new learning in any of the identified areas and a score of " 3 " highlighted new learning in all outlined areas. Each score was supported by relevant comments made by participants.

Next, the rubric was used to evaluate participants' responses to the question, "What were your general impressions of the teleconsultation experience?" Participants' responses were ranked 0-3 based on overall negative and positive feedback of the experience. A score of " 0 " indicated that feedback related to the teleconsultation experience was only negative with the participant reporting maximum levels of frustration, disappointment, etc. A score of " 3 " was given when the participant only had positive feedback related to the teleconsultation experience. Again, each score was supported by comments shared during presentations.

Throughout the coding process, data was analyzed for emerging concepts. Participants' comments and feedback aided in theme development. After each investigator completed the data analysis process including coding and theme formation, measures were used to increase the trustworthiness of findings. Data was triangulated by reviewing each other's coding and through member checking with the participants to ensure accuracy.

\section{RESULTS}

\section{REPORTED NEW LEARNING}

The majority of participants (85.7\%) explicitly reported new learning in at least one of the three outcome areas of occupational therapy (OT) process, culture, and technology. Most new learning was reported in the area of technology $(71 \%)$, followed by OT process $(57 \%)$, and then culture (43\%) (Figure 1).

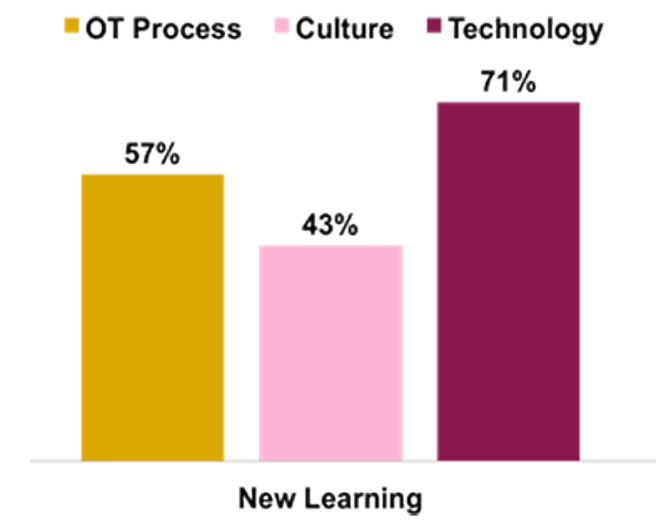

Figure 1. Reported new learning in evaluated outcome areas. 
GENERAL IMPRESSIONS OF THE TELECONSULTATION EXPERIENCE

Participant feedback indicated the following: no participants reported only negative feedback, $20 \%$ provided mostly negative feedback, $30 \%$ provided mixed feedback (both positive and negative), and $50 \%$ provided only positive feedback with regard to participating in the Teleconsultation project (Figure 2). countries that I was not aware of myself like Internet complications and scheduled power outages. It was an eye-opening experience and nice to help the people who may have not been able to get this help... It gave me some insight of the challenges and benefits of working with telehealth in order to treat patients across cultures.

In addition to gaining an overall appreciation for cultural differences, participants spoke about specific opportunities for learning and challenges related to factors such as lifestyle differences and language barriers.

It was hard to schedule due to their laid-back lifestyle. We are always go go go and they are very laid-back, so it was interesting to see the two different cultures in that sense.

It seemed like a very important goal to our client was being able to walk outside for long distances since they walk everywhere in Guatemala.

The language barrier made it difficult for us to know if they understood what we were saying and we had to problem-solve ways to get them the information. For example, we found this nice website that will translate information about strokes into different languages, so we ended up using that for our home exercise program.

Figure 2. General impressions of the teleconsultation experience.

\section{THEMATIC DEVELOPMENT}

Following qualitative data analysis, outcome themes included: (a) increased understanding of and challenges to working with people of different cultures, (b) need for adaptability and flexibility as a practicing clinician, and (c) emerging role of technology in occupational therapy.

\section{INCREASED UNDERSTANDING OF AWARENESS} OF AND CHALLENGES TO WORKING WITH PEOPLE OF DIFFERENT CULTURES

Before participating in the teleconsultative experience, study participants had varied amounts of interaction with individuals from developing countries. Prior to this assignment, many of the participants' clinical observation sites had minimal diversity and with the exception of a few participants who reported having family located internationally, interaction with individuals from different cultural backgrounds was limited. During their presentations, participants spoke about appreciating an opportunity to work with and learn from individuals with different cultural backgrounds.

This experience really helped increase my understanding of clients with different backgrounds. There are some ethnocentric things about other
Some participants made comments that demonstrated insight into relevance of this assignment beyond working with people in Guatemala. Certain remarks indicated that although this experience related specifically to individuals in a developing country, many of the cultural concepts will directly relate to their future practice as occupational therapy clinicians regardless of location.

The language barrier was frustrating, but a good experience because in the future we will have to work with these sorts of barriers.

It helped me understand how a culture plays a part in occupation. It gave me an understanding of cultural barriers that can impact occupational therapy performance like communication and lifestyle differences.

\section{NEED FOR ADAPTABILITY AND FLEXIBILITY AS PRACTICING CLINICIANS}

Throughout their MSOT education, students are provided with insight into the need for adaptability and flexibility as occupational therapists. Anecdotal stories told by educators as well as supporting texts and literature are shared with students to prepare them for challenges they will face in their careers. Learning in certain areas is best accomplished through experiential opportunities as supported by development of this particular theme. 
The assignment (Appendix A) charged students with a task of wholly managing a client including execution of logistical tasks such as scheduling. For many participants, this was the first time they were required to be involved with this aspect of client care. Although this yielded frustration for many, influencing some's overall impression of teleconsultation, participants consistently reported on the relevance of scheduling and compliance in regards to the occupational therapy process.

It is really important to be flexible. It is also important to learn how difficult scheduling can be and how difficult language barriers can be. The difficulties with scheduling are going to be general to our career so it helps us to understand the possible conflicts that may arise.

I thought it was interesting to see the reality of therapy where your patients will cancel or arrive late.

The use of technology for long-distance therapy and the nature of the experience requiring the group to adapt will teach us how to adapt when we are in the world of actual therapists.

\section{EMERGING ROLE OF TECHNOLOGY IN} OCCUPATIONAL THERAPY

Within an area in which $71 \%$ of participants reported new learning (Figure 1), the awareness of the emerging role of technology in occupational therapy was a salient theme that emerged. Prior to participation in the teleconsultation experience, students had minimal education in the provision of remote services to clients through the use of technology. To prepare them for this assignment, the course instructor provided them with resources and a brief review of telehealth, but no students had observed or participated in experiential learning involving telehealth prior to this project. Thematic analysis revealed that although participants were taught basic concepts about the use of technology in provision of healthcare, many developed a greater understanding after participation in this experience.

It was really nice to be able to use telehealth because this is something that is going to be up and coming and is already in our field. I expect it to be much more prevalent as the years go on and technology continues to emerge. I think it was great to have a hands-on experience and to be able to do this. I'd assume that most occupational therapy students don't always get this opportunity.

It increased my overall impression of where our field and overall medical profession can be heading. The VSee (technology) is already set up so this is not something that is futuristic. It is already on the forefront.

In addition to a general awareness of the relevance of telehealth in occupational therapy practice, participants also indicated an understanding of how remote care differs from in-person treatment sessions.

When in fieldwork, you always had hands-on opportunities and this was different because the patients were across the country and you didn't have the chance to touch them and it was just a very different experience that l've never had.

As with other themes, participants also related this experience to services that can be provided locally, not just internationally.

I think telehealth is a great thing, not just for other countries but for me, too. I am from a poor urban area and this really speaks to me because there are many people in our own country that don't have access to OT and they don't know what OT is. Even though this assignment was abroad, there may be certain cities and other areas in our own country that may not know what OT is. Again, it is important to advocate within our own state and country.

\section{DISCUSSION}

This study shows students' reported learning and overall feedback regarding a teleconsultation experience embedded into an MSOT curriculum. Although there were limitations in both the program and the study design, new learning was evident. One interesting finding of this study was that although student groups averaged only one, thirty minute visit with their client, the majority of participants reported new learning in at least one outcome area. During coding, it was evident that the participants who reported no new learning were also the ones who reported mostly negative feedback related to the teleconsultation experience. This correlation suggests that participant attitude and perception influenced perceived learning. Many participants were able to relate both positive and negative aspects of this experience to its relevance in their future careers. For example, challenges related to scheduling, client compliance, and language barriers were frequently noted as potential future experiences they will encounter when they are practicing occupational therapists. Additionally, several participants noted that although there were aspects of teleconsultation that could be frustrating (no "hands on" care, technical difficulties, etc.), the emerging role of technology in the occupational therapy profession made this a relevant and enriching learning experience. Participants who were unable to connect the classroom assignment to occupational therapy practice were those who had mostly negative feedback related to this experience.

Despite some meaningful results that will contribute to program development, there are many limitations and indications for future studies related to teleconsultation and pedagogy. 


\section{LIMITATIONS IN TELECONSULTATION IMPLEMENTATION}

There were several technological and cultural barriers related to the (Guatemalan) rehabilitation clinic. The primary contact at the clinic was a recipient of services and did not have resources needed for consistent scheduling and communication. The reliance on technology for communication posed challenges in all stages of the experience, not just service provision. Researchers relied on VSee videoconferencing, email, and WhatsApp, a mobile device messaging application, for communication. Oftentimes, if the Internet was not available in Guatemala, the contact would use WhatsApp to message researchers, but participants were not privy to this resource. Therefore, communication became a barrier not just between clients and (student) clinicians, but also between program developers.

In addition to technological difficulties, individuals who were identified as potential service recipients were oftentimes noncompliant with appointments. There were several missed sessions, often due to accessibility and transportation issues identified in the preliminary study. Oftentimes, clients would arrive for consultation 2-3 hours after the identified appointment time when participants were no longer available. Clients would become frustrated that they traveled for therapy services they were unable to receive; participants would share this frustration especially with the intense course scheduling of their graduate programming.

As identified in the results section, many participants were frustrated with the language barrier. Only one of the 21 participants spoke Spanish, the language of the clients; the language barrier challenged both (student) clinicians and clients. Because at times technical difficulties yielded only audio availability with no video, this posed further challenges to adapting to language barriers.

\section{SUGgestions FOR Future PROGRAM PLANNING}

There are several modifications that could be implemented to continue and improve upon this pilot program. One modification could be to identify an international partner who has adequate resources to support the experiential learning assignment; however, the institution would like to continue its relationship with the current partner. The Guatemala site is a grassroots initiative that was started by an individual who sustained a spinal cord injury. Frustrated by the lack of services available to him, he turned his own home into a rehabilitation center where he provides services to people throughout the country. With the exception of a physical therapy student, he has no professional services available to him. Therefore, the teleconsultation program not only provides students with an enriching learning experience, but also provides this clinic with rehabilitation services they would otherwise not receive.

To address the issues of scheduling and lack of resources at the rehabilitation clinic, the MSOT program intends on implementing an "open hours" clinic in lieu of scheduling clients to specific times. With this method, (student) clinicians will be available during regular hours each week and any clients who arrive at the clinic will be offered consultation in collaboration with faculty mentors.

Regarding the language barrier, it is essential to increase the availability of interpreters. The highlighted MSOT program is part of a larger school of health sciences with Bachelor, Master, and Doctoral level students studying various health care fields. Programmers are hoping to take advantage of these resources in order to identify bilingual students who would be interested in participating in this teleconsultation program. Not only will this address a widely recognized challenge of the program and increase communication between the service providers and recipients, but will also initiate an interprofessional education initiative with potential for growth.

\section{LIMITATIONS IN STUDY DESIGN}

Although this study served as an initial review of a pilot program and yielded important results, there are noteworthy limitations. First, because of the small program size, the sample of 21 students who worked in seven smaller groups does not yield highly generalizable results. Additionally, as this study was linked to a course assignment, there are several potential biases related to participant feedback. Data was obtained from student presentations and although the primary researcher was not the course instructor, this may have influenced participant feedback.

Using a qualitative design, this study lacked standardized outcome measures. Despite using a rubric for coding, the qualitative design yielded different student/researcher perceptions of learning. Per the primary investigator who is an instructor in the program, participants demonstrated high levels of integration of knowledge from occupational therapy course(s) with the teleconsultation assignment, as made evident through use of terminology and clinical reasoning related to choices of assessment tools, treatment techniques, discharge plans/recommendation, etc. Due to the study design, it was difficult to determine what new learning occurred as a result of this experience; however, the high level integration of knowledge deemed this assignment to be an effective learning experience. 


\section{SUGGESTIONS FOR FUTURE RESEARCH}

Once the teleconsultation program is modified based on student and instructor feedback, there are potential areas for more rigorous scholarship to assess program outcomes. It is recommended that future research designs utilize standardized tools with quantitative analyses of new learning areas indicated in this study.

\section{CONCLUSION}

The findings in this study contribute to expanding knowledge of the implementation of telehealth and teleconsultation into occupational therapy programs. This study demonstrates that integrating experiential international teleconsultation opportunities into allied health curricula facilitates new learning in the areas of the therapy process, culture, and technology. The experiences of participants in this program highlight the importance of using creative teaching methods and assignments in order to address accreditation standards and learning outcomes. Occupational therapy academicians should continue to implement these types of programs into curricula and conduct related research in order to promote not only student learning, but also to advance the use of technology in occupational therapy practice.

\section{REFERENCES}

1. American Occupational Therapy Association. (2006). AOTA's Centennial Vision. Retrieved from http://www. aota.org/News/Centennial/Background/36516.aspx.

2. American Occupational Therapy Association. (2013). Telehealth [Position paper]. American Journal of Occupational Therapy, 67, S69-S90. doi:10.5014/ ajot.2013.67S69

3. American Occupational Therapy Association. (2013). 2011 Accreditation Council for Occupational Therapy Education (ACOTE $®$ ) Standards and Interpretive Guide: December 2013 Interpretative Guide Version. Retrieved from http://www.aota.org/-/media/Corporate/Files/ EducationCareers/Accredit/Draft-Standards/2011Standards-and-Interpretive-Guide-August-2013.pdf

4. Barlow, I.G, Liu, L, \& Sekulic, A. (2009). Wheelchair seating assessment and intervention: A comparison between telerehabilitation and face-to-face service. International Journal of Telerehabilitation, 1(1), 17-28. doi: 10.5195/ ijt.2009.868. Retrieved from http://telerehab.pitt.edu/ojs/ index.php/Telerehab/article/view/868

5. Bulik, R. \& Shokar, G.S. (2010). Integrating telemedicine instruction into the curriculum: Expanding student perspectives of the scope of clinical practice. Journal of Telemedicine and Telecare, 16, 355-358. doi: 10.1258/ jtt.2010.090910
6. Cason, J. (2009). A pilot telerehabilitation program: Delivering early intervention services to rural families. International Journal of Telerehabilitation, 1, 29-38. http:// dx.doi.org/10.5195/ijt.2009.6007

7. Cason, J. (2011). Telerehabilitation: An adjunct service delivery model for early intervention services. International Journal of Telerehabilitation, 3(1), 19-28. http://dx.doi. org/10.5195/ijt.2011.6071

8. Eleazar, C., Campisi, M., \& Foti, M. (2013, October 27). Facilitators and barriers to living with a disability in Guatemala. Poster session presented at the New Jersey Occupational Therapy Association Annual Conference, Burlington, New Jersey.

9. Fries, K.S., Bowers, D.M., Gross, M., \& Frost, L. (2013). Service learning in Guatemala: Using qualitative content analysis to explore an interdisciplinary learning experience among students in health care professional programs. Journal of Multidisciplinary Healthcare, 6, 4552. doi: 10.2147/JMDH.S35867

10. Hoffmann, T., \& Russell, T. (2008). Pre-admission orthopaedic occupational therapy home visits conducted using the internet. Journal of Telemedicine and Telecare, 14, 83-87.

11. Hoffmann, T., Russell, T., Thompson, L., Vincent, A., \& Nelson, M. (2008). Using the internet to assess activities of daily living and hand function in people with Parkinson's disease. NeuroRehabilitation, 23, 253-261.

12. Jacobs, K., Blanchard, B., \& Baker, N. (2012). Telehealth and ergonomics: A pilot study. Technology and Health Care, 20, 445-458.

13. Lee, A., Parmanto, B., Saptono, A., Pulantara, W., Sargent, B., \& Arroyo, J.L.F. (2012). The VISYTER telerehabilitation system for globalizing physical therapy consultation: Issues and challenges for telehealth implementation. Journal of Physical Therapy Education, 26(1), 90-96.

14. Lee, A., \& Norton E. (2009). Use of telerehabilitation to address sustainability of international service learning in Mexico: Pilot case study and lessons learned. HPA Resource, 9,1- 4.

15. Liu, L. \& Miyazaki, M. (2000). Telerehabilitation at the University of Alberta. Journal of Telemedicine and Telecare, 6(2), 47-49. doi: 10.1037/0735-7028.36.2.173

16. Miller, T.W., Miller, J.M., Burton, D., Sprang, R., \& Adams, J. (2003). Telehealth: A model for clinical supervision in allied health. Internet Journal of Allied Health Sciences and Practice, 1(2), 1-8. Retrieved from http://ijahsp.nova. edu/articles/1vol2/miller.pdf

17. Schein, R.M., Schmeler, M.R., Holm, M.B., Pramuka, M., Saptono, A., \& Brienza, D.M. (2011). Telerehabilitation assessment using the Functioning Everyday with a Wheelchair-Capacity instrument. Journal of Rehabilitation Research \& Development, 48, 115-124.

18. Sanford, J., Hoenig, H., Griffiths, P., Butterfield, T., Richardson, P., \& Hargraves, K. (2007). A comparison of televideo and traditional in-home rehabilitation in mobility impaired older adults. Physical and Occupational Therapy in Geriatrics, 25(3), 1-18. 


\section{APPENDiX A. GUIDELINES FOR GUATEMALA TELECONSUlTATION PROJECT AND PRESENTATION}

\section{THE CONCEPT}

The ACOTE Standards for Master's Level Occupational Therapy Programs (2011) were initiated in July 2013. There is a new standard that states that students should "Demonstrate an understanding of the use of technology to support performance, participation, health and well-being. This technology may include, but is not limited to, electronic documentation systems, distance communication, virtual environments, and telehealth technology. (B.1.8)". This assignment will specifically address this standard. However, there are several other Standards that will be addressed during your participation in this assignment (see syllabus).

\section{THE PROJECT}

1. Groups of 3 students will meet once/week with the client.

2. You will do a total of 4-6 visits with the client.

a. The number of visits will depend on the client's needs.

b. Visits can last from 5 minutes to an hour, as necessary.

c. Visits MUST occur in an on campus occupational therapy space. YOU CANNOT MEET WITH THE CLIENT IN YOUR OWN HOMES, OFF CAMPUS, OR ANY AREA NOT LISTED ABOVE.

d. Confidentiality MUST be maintained. Therefore, only students in our class, your mentors, and those designated as translators can know the details of your client.

3. You will make your own appointments with the client.

a. The first visit will be arranged with me and our primary Guatemala contact, but visits after that need to be made at the end of your visit.

b. Keep in mind that there is only 1 computer at the facility in Guatemala so 2 groups cannot be meeting at the same time. You may need to speak with the other groups to see when they have scheduled visits.

c. Translation may be necessary for some clients.

4. You can use any evaluation tool you wish, but might I suggest the COPM since you should be familiar with this tool by the time you set up your first visit.

5. You will develop activities based on your client's needs

6. You may need to send documents to the clients for exercises, etc. You can achieve this through VSee and likely through e-mail.

7. After each visit, you need to meet with your mentor to discuss what you did with the client and what the plan for the next visit is.

a. Keep in mind that your mentors work so you need to be flexible in scheduling meetings with them.

b. All group members should be available for those meetings, but missing 1 meeting is OK.

c. Meetings can be done in person, via phone, via Skype or other method.

\section{THE PRESENTATION}

Each group will develop a presentation that discusses the following:

1. Background Information on your client (Evaluation)
a. Name
b. Age
c. Diagnosis
d. Presenting Problems/Complaints-based on your evaluation
e. Client Goals-based on your evaluation

f. Intervention Goals-use correct format

2. Recommendations/Plan (Implementation)

a. Number of times seen and why? Justify your LOS.

b. Activities Recommended

i. Why did you choose the activities you chose?

ii. Did you make any changes to your plan and activities? Why?

iii.What plan did you leave your client with? That is, what is the HEP you developed for them , if any?

3. Outcomes (Discharge and Outcomes)

i. Were the goals achieved? Why? Why not?

ii. What would you do differently next time?

iii. What were your general impressions of the telehealth experience? 


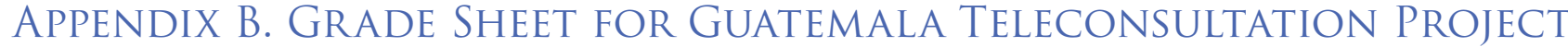 AND PRESENTATION}

Background Information on your client (Evaluation)

- Name

- Age

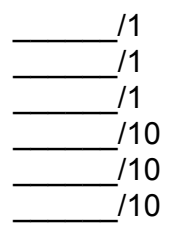

- Diagnosis

- Presenting Problems/Complaints-based on your evaluation 0

- Client Goals-based on your evaluation

- Intervention Goals-use correct format

Recommendations/Plan (Implementation)

- Number of times seen and why? Justify your LOS.

17

- Activities Recommended

$\checkmark \quad$ Why did you choose the activities you chose?

$\checkmark$ Did you make any changes to your plan and activities? Why?

$\checkmark$ What plan did you leave your client with? That is, what is the HEP you developed for them, if any, for the activities you provided?

Group Member 1

Group Member 2

Group Member 3

Outcomes (Discharge and Outcomes)

- Were the goals achieved? Why? Why not?

- What would you do differently next time?

- What were your general impressions of the telehealth experience?

Group Member 1

Group Member 2 18

Group Member 3 18

Grade from your peer evaluation: 110

TOTAL: 
